\title{
Alcoholic liver disease
}

\author{
Kevin Walsh, Graeme Alexander
}

\begin{abstract}
Alcohol is a major cause of liver cirrhosis in the Western world and accounts for the majority of cases of liver cirrhosis seen in district general hospitals in the UK. The three most widely recognised forms of alcoholic liver disease are alcoholic fatty liver (steatosis), acute alcoholic hepatitis, and alcoholic cirrhosis. The exact pathogenesis of alcoholic liver injury is still not clear but immune mediated and free radical hepatic injury are thought to be important. There is increasing interest in genetic factors predisposing to hepatic injury in susceptible individuals. Diagnosis is based on accurate history, raised serum markers such as $\gamma$-glutamyltransferase, mean corpuscular volume, and IgA and liver histology when obtainable. Abstinence is the most important aspect of treatment. Newer drugs such as acamprosate and naltrexone are used to reduce alcohol craving. Vitamin supplements and nutrition are vital while corticosteroids have a role in acute alcoholic hepatitis where there is no evidence of gastrointestinal haemorrhage or sepsis. Liver transplantation has excellent results in abstinent patients with end stage liver disease but there are concerns about recidivism after transplant.
\end{abstract}

(Postgrad Med f 2000;76:280-286)

Keywords: cirrhosis; liver disease; alcohol

Epidemiology

Alcoholic liver disease (ALD) is one of the major medical complications of alcohol abuse. Alcohol is the major cause of liver cirrhosis in the Western world, with schistosomial infection being the major cause of portal hypertension in the developing countries. Alcohol accounts for $80 \%$ of all liver cirrhosis cases seen in district general hospitals in the UK. Alcoholic cirrhosis is increasingly seen in countries such as Japan and India which traditionally had a low prevalence of the disease.

The safe limits for alcohol intake are controversial. Guidelines recommended by the Royal College of Physicians advise a weekly limit of 21 units $(210 \mathrm{~g})$ of alcohol in men and 14 units in women. The 1994 Office of Population Censuses and Surveys' General Household Survey found that $27 \%$ of men and $13 \%$ of women in the UK were exceeding these limits. A recent prospective Italian study in 6534 subjects showed the risk threshold for developing ALD is $30 \mathrm{~g}$ ethanol/day and this risk increases with increasing daily intake. ${ }^{1}$ Women had greater susceptibility to ALD in the range of 3-8 drinks daily. A previous study in 13285
Box 1: Safe limits for alcohol intake

- Males $=21$ units per week (1 unit = glass or half pint)

- Females $=14$ units per week

Box 2: Factors increasing susceptibility to ALD

- Lifetime intake of alcohol

- Female sex

- Genetic factors

- Drinking without food

- Binge drinking

- High concentration alcoholic drinks-for example, spirits

- Drinking multiple different alcoholic beverages

Danish subjects found that ALD was increased above a threshold of 7-13 drinks per week in females and 14-27 drinks in males. ${ }^{2}$ There was a steep dose dependent increase in ALD risk above this threshold with increasing alcohol intake. Women had greater susceptibility to ALD at any given level of intake. Kwo et al have recently shown that men and women have equivalent alcohol elimination rates. ${ }^{3}$ The gender differences in susceptibility to liver injury may be due to pharmacokinetic reasons such as differences in ethanol absorption or alternatively differences in the response of the liver to alcohol induced injury such as that caused by oxidative by-products of ethanol metabolism in the liver.

The pattern of drinking was also important as ALD is increased in those who drink without accompanying food and also in those who drink multiple different alcoholic beverages. ${ }^{1}$ It is well known that food delays gastric emptying and intestinal absorption of alcohol and thus intake of food before drinking will decrease the rise of blood alcohol concentrations. The absorption of alcohol is lower when consuming low concentration beverages such as beer compared with high concentration spirits.

A recent study in 12687 subjects showed that coffee consumption protected males from the induction of $\gamma$-glutamyltransferase by alcohol and may possibly protect against liver cell damage caused by alcohol. ${ }^{4}$

Spectrum of liver disease

The three most widely recognised forms of ALD are alcoholic fatty liver (steatosis), acute alcoholic hepatitis, and alcoholic
Submitted 3 June 1999 Accepted 6 September 1999 
cirrhosis. At least $80 \%$ of heavy drinkers develop steatosis, 10\%-35\% develop alcoholic hepatitis, and approximately $10 \%$ will develop cirrhosis. $^{5}$

ALCOHOLIC FATTY LIVER (STEATOSIS)

Steatosis is invariable if consumption exceeds $80 \mathrm{~g}$ of alcohol per day. A large proportion of the cytoplasm of affected hepatocytes is occupied by a single large triglyceride occlusion but liver function is often normal. It is reversible with abstinence but may progress to cirrhosis if excess alcohol intake persists. ${ }^{6}$ Deaths due directly to fatty liver are rare and are usually caused by acute liver failure or fat embolism.

\section{ACUTE ALCOHOLIC HEPATITIS}

In alcoholic hepatitis, hepatocyte ballooning occurs due to increased intracellular water accumulation. Mallory's hyaline bodies are perinuclear eosinophilic inclusion bodies and are probably condensed and disorganised fragments of the cytoskeletal framework of the hepatocyte. They are not specific for alcoholic hepatitis as they are also seen in Wilson's disease and primary biliary cirrhosis. It has been estimated that 15-20 years of excessive drinking is necessary to develop alcoholic hepatitis. There is prominent cholestasis. It is more severe in females and also in Northern Europeans and is unrelated to pattern of drinking or type of alcohol drink. High mortality rates are seen $(30 \%-60 \%)$. Patients often deteriorate after hospital admission despite abstinence. This latter phenomenon may be due to reperfusion liver injury.

\section{ALCOHOLIC CIRRHOSIS}

Cirrhosis is the most severe form of alcoholic liver injury and is usually of the micronodular type. The risk is increased in continuous drinkers compared to binge drinkers. Collagen deposition can be pericellular, in the space of Disse, and around central veins (central hyaline sclerosis). The latter is often associated with rapid progression to cirrhosis and commoner and more severe in women. Collagen bridges eventually develop between central veins and portal tracts isolating groups of hepatocytes which form the regeneration nodules. Survival for patients is $60 \%-70 \%$ at one year and $35 \%-$ $50 \%$ at five years.

\section{Pathogenesis}

There are a number of hypotheses regarding the pathogenesis of alcoholic liver injury.

Ethanol metabolism usually takes place in the mitochondria. Ethanol is oxidised to acetaldehyde by alcohol dehydrogenase, which in turn is oxidised to acetate by acetaldehyde dehydrogenase. These oxidation reactions are associated with the formation of $\mathrm{NADH}$ and NAD and alter the redox state of the cell. This has harmful effects on lipid and carbohydrate metabolism-for example, steatosis. In habitual drinkers, a microsomal mixed function oxidase, the microsomal ethanol oxidising
Box 3: Major classification of ALD

- Alcoholic fatty liver (steatosis)

- Acute alcoholic hepatitis

- Alcoholic cirrhosis

Box 4: Histological features of ALD

- Steatosis (fatty liver)

- Mallory's hyaline bodies

- Cholestasis

- Liver fibrosis

- Micronodular cirrhosis

system, is increased by enzyme induction and is also responsible for production of acetaldehyde.

Acetaldehyde is increased in zone 3 (also the maximal site of action of alcohol dehydrogenase). This is also the site of the terminal veins making this zone the most hypoxic and therefore highly susceptible to hypoxic injury. Oxygen derived free radicals may cause direct hepatocyte injury by lipid peroxidation.

Acetaldehyde binds covalently to proteins forming adducts that are antigenic. Humans and animals exposed to long term alcohol excess develop persistent circulating antibodies that recognise acetaldehyde protein adducts. ${ }^{7}$ Acetaldehyde modified self proteins may serve as neoantigens, initiating harmful humoral and/or cellular immune responses, which leads to tissue injury. ${ }^{8}$ The expression of the proinflammatory cytokines, tumour necrosis factor-alpha (TNF- $\alpha)$, transforming growth factor-beta (TGF- $\beta$ ), interleukin (IL)$1 \beta$, and IL- 6 are increased in alcoholic liver injury while the anti-inflammatory cytokine, IL-4 is decreased. ${ }^{9}$ These cytokines stimulate stellate cells which produce collagen leading to liver fibrosis. A study by Neuman et al suggests that it is TNF- $\alpha$ that is the main candidate accounting for toxicity as addition of an equivalent amount of IL-6 seen in ALD did not produce injury in a normal rat liver whereas equivalent amounts of $\mathrm{TNF}-\alpha$ did produce injury. ${ }^{10}$ Removal of toxic reactive oxygen species is achieved via three major antioxidant enzymes: catalase, superoxide dismutase, and glutathione peroxidase. The generation of high concentrations of free radicals during the metabolism of alcohol may exceed the capacity of the antioxidant defence mechanisms and contribute to the development of alcohol induced liver injury. Polavarapu et al have shown that in rats with alcohol induced injury, these antioxidant enzymes are reduced and levels are inversely correlated with severity of liver injury. ${ }^{11}$ Alcohol reduces alkaline protease activity in rats resulting in failure to clear hepatocytes of oxidatively damaged proteins. ${ }^{12}$ This results in oxidative liver damage.

Soluble fatty acid synthase (FAS) and FAS ligand are increased in patients with 
acute alcoholic hepatitis and alcoholic cirrhosis while they are normal in chronic hepatitis C. ${ }^{13}$ These soluble mediators could be produced in hepatocytes, Kuppfer cells, or polymorphonuclear cells in the liver. Their exact role in alcoholic liver injury needs to be determined.

Twin studies suggest a genetic component to disease susceptibility. ${ }^{14}$ In white people, associations between ALD risk and polymorphisms of the genes encoding the cytochrome $P-450$ CYP2E $1{ }^{15}$ and TNF- $\alpha^{16}$ have been shown. The frequency of an alcohol dehydrogenase 3 allele has been found to differ in alcohol related liver disease and controls. ${ }^{17}$ IL-10 polymorphism is associated with an increased risk $(\times 1.8)$ of ALD.

Hepatitis C viraemia and alcohol have an additive effect on the risk of liver injury. ${ }^{18}$ Alcohol may impair the immune system's response to the virus or increased hepatic iron secondary to alcohol may modify the clinical course of hepatitis $\mathrm{C}$ virus.

There is significant iron deposition in alcoholic livers. ${ }^{19}$ A recent study found no link between simple heterozygotes of either HFE gene and risk of ALD. ${ }^{20}$ Comparing 257 patients with ALD and 117 healthy controls, $15.7 \%$ of fibrotic/cirrhotic patients were C282Y heterozygote compared with $13.7 \%$ of controls. Of chromosomes without the $\mathrm{C} 282 \mathrm{Y}$ mutation, $15.4 \%$ of ALD and $16.6 \%$ of controls were H63D heterozygote. Significant iron staining was found in $6 / 23 \mathrm{C} 282 \mathrm{Y}$ heterozygotes versus $4 / 26 \mathrm{H} 63 \mathrm{D}$ heterozygotes versus $4 / 23$ controls. Simple heterozygotes are not more susceptible to developing iron catalysed oxidative stress during ethanol consumption. Patients with ALD have increased iron accumulation, independent of HFE genotype. This may be because of folic acid deficiency increasing iron absorption. ${ }^{21}$ Oxidative stress increases the activity of iron regulatory proteins which bind to iron response elements in the messenger RNAs of iron regulating genes such as the transferrin receptor and ferritin. ${ }^{22}$ This results in an increase in free iron in the hepatocyte. Hepatic siderosis in an alcoholic may identify those most susceptible to oxidative stressbecause of genetic or environmental reasons. There is an inverse correlation between liver iron staining and survival in alcoholic cirrhosis. $^{23}$

Alcohol increases portal blood flow and also hepatic vascular resistance leading to an increase in portal pressure and collateral blood flow. ${ }^{24}$ This increases the risk of variceal bleeding in patients with alcoholic cirrhosis and portal hypertension.

\section{Clinical presentation}

Alcohol can cause significant liver damage without producing any symptoms or signs of liver disease. Many people are diagnosed when they have routine liver function tests as part of a medical check-up. Patients may present with non-specific features such as nausea, vomiting, abdominal discomfort, or diarrhoea. Patients with advanced liver disease may present with

\section{Box 5: Putative pathogenic} mechanisms

- Lipid peroxidation

- Reduced antioxidant defences

- Immunological

- Iron deposition
Box 6: Clinical features
- Nausea
- Abdominal discomfort
- Diarrhoea
- Jaundice
- Ascites
- Encephalopathy
- Upper gastrointestinal bleeding

jaundice, ascites, encephalopathy, or upper gastrointestinal bleeding.

ALD may be detected when patients present voluntarily for detoxification. Patients may present with other sequelae of alcoholism such as pneumonia, rib fractures, or head injury. Liver damage may be detected when patients present with alcoholic damage to other organs such as the pancreas, heart, brain, and peripheral nerves.

Patients with fatty liver usually look well and often have only mild hepatomegaly with no other stigmata of chronic liver disease.

In alcoholic hepatitis patients usually look very unwell, are malnourished, and often pyrexial. Patients are often deeply jaundiced and cutaneous features of chronic liver disease are common. There is often marked hepatomegaly which is tender on palpation. Hepatic bruits may be heard. Splenomegaly is uncommon. Ascites and hepatic encephalopathy are common but varices are not common. Upper gastrointestinal bleeding does occur but is usually due to gastric erosions or peptic ulceration on a background of coagulopathy. Infections are very common during the illness.

The clinical features of alcoholic cirrhosis are similar to non-alcoholic cirrhosis. It is thought that spider telangiectasia, parotid enlargement, gynaecomastia, and hepatomegaly are more common in alcoholic cirrhosis.

\section{Investigations}

The most important part of the diagnostic work-up is obtaining a reliable history of prolonged alcohol abuse. Patients are often not forthcoming until they gain the trust of their doctor and so their general practitioner may be in the best position to make the diagnosis. Various questionnaires such as the CAGE test are useful in screening for alcohol abuse. A collateral history from spouses, family members, and friends is very important. 
The two commonest laboratory indices in detecting excessive alcohol consumption are a raised serum $\gamma$-glutamyltransferase and mean corpuscular volume. Individually these test have a sensitivity of $30 \%-40 \%$ but combining the tests is much more informative. A moderately raised $\gamma$-glutamyltransferase can be found in non-alcoholic fatty liver and also in patients taking drugs which induce hepatic enzymes such as phenytoin. An isolated raised $\gamma$-glutamyltransferase in a heavy drinker with otherwise normal liver function tests is usually associated with only mild histological liver damage which is reversible. Many clinicians therefore feel a liver biopsy to assess liver injury is unnecessary in this situation.

Serum carbohydrate deficient transferrin is a specific and sensitive test of alcoholism but is not widely available and many feel that a combination of $\gamma$-glutamyltransferase and mean corpuscular volume is just as useful.

Serum transaminase levels are often not greatly raised in ALD and values greater than five times the upper limit of the normal reference range should lead to consideration of other diagnoses such as viral or autoimmune hepatitis. Hyperbilirubinaemia reflects the severity of the hepatitis and is greatly increased in alcoholic hepatitis.

Prolongation of the prothrombin time and hypoalbuminaemia reflect poor hepatic synthetic function.

A score based on prothrombin time (seconds) and serum bilirubin ( $\mathrm{mmol} / \mathrm{l})$ is used to determine short term prognosis in alcoholic hepatitis. ${ }^{25}$ The discriminant function (DF) is calculated from the equation $\mathrm{DF}=4.6$ (prothrombin time - control time) + serum bilirubin/17.1. A score $>32$ denotes severe disease with a two month mortality of $50 \%$ and these patients should be given a trial of corticosteroids.

Leucocytosis is common in alcoholic hepatitis as is thrombocytopenia, which occurs secondary to direct alcohol toxicity or to hypersplenism found in portal hypertension. Electrolyte abnormalities commonly found are hyponatraemia and hypokalaemia and less commonly hypocalcaemia and hypomagnesaemia.

Ultrasound scanning can reveal the presence of fatty liver and hepatitis through changes in the reflectivity of the liver parenchyma. An irregular shrunken liver suggests severe cirrhosis while additional pointers to liver disease such as ascites, varices, and splenomegaly can be identified. Doppler studies can demonstrate changes in hepatic artery and portal vein flow. Newer radiological tools such as magnetic resonance spectroscopy are being evaluated. ${ }^{26}$

A number of serum markers of fibrosis have been shown to reflect severity of alcoholic liver injury such as procollagen III propeptide, laminin, and type IV collagen. However they lack enough sensitivity and specificity to be used in individual cases.

Liver biopsy is necessary to confirm the extent of liver injury and to provide a prognostic guide. The coagulopathy accompanying alcoholic hepatitis and cirrhosis makes this a

\section{Box 7: CAGE questionnaire}

C. Have you ever felt the need to cut down your drinking?

A. Have you ever felt annoyed by criticism of your drinking?

G. Have you ever felt guilty about your drinking?

E. Have you ever taken a drink (eye opener) first thing in the morning?

Score 1 for each positive response. A score of 2 or more suggests alcohol excess.

\section{Box 8: Investigations}

- $\gamma$-Glutamyltransferase

- Mean corpuscular volume

- Carbohydrate deficient transferrin

- Bilirubin (markedly raised in acute alcoholic hepatitis)

- Serum transaminases: if greater than five times normal suspect other liver pathology

- Prothrombin time

- Albumin

- Serum fibrosis markers: procollagen III propeptide, laminin, type IV collagen

- Liver ultrasound: also detect splenomegaly and ascites

- Liver biopsy

riskier procedure, which may necessitate using the transjugular venous route for performing the biopsy.

Portal pressure measurements taken at hepatic venography may be superior to the Child-Pugh score in predicting mortality and bleeding risk in patients with alcoholic cirrhosis but no previous bleeding. ${ }^{27}$

\section{Treatment}

The most important aspect of treatment is abstinence from alcohol. This should be encouraged with help from the patient's family and friends, psychiatric intervention, and also support groups such as Alcoholics Anonymous. Despite these measures, many patients continue to abuse alcohol and this has led to great interest in pharmacological agents to treat alcohol dependence. The opiate antagonist, naltrexone has been licensed in the USA for treatment of alcoholism since 1994. This is used in patients who are actively drinking, where it has been shown to reduce craving and the level of drinking over several months. It has no role in enhancing abstinence. A newer opiate antagonist, nalmefene, has the advantage of not being toxic to the liver and having no first-pass metabolism but data are insufficient at present to recommend its clinical use. Acamprosate is the other agent which has also been shown to reduce drink frequency but its effect on abstinence is unclear. The mode of 
action of acamprosate is unknown but it appears to interact with glutamate receptors and calcium channels. A recent meta-analysis has confirmed the clinical benefits of naltrexone and acamprosate but has cast doubt on the benefits of disulfiram which has been traditionally used to promote abstinence. ${ }^{28}$ The meta-analysis also showed that serotonergic drugs and lithium have no clinical benefit in managing alcohol misuse.

Vitamin B deficiency is common in ALD, in particular thiamine, folate, pyridoxine, and riboflavine. Parenteral vitamin B supplementation is preferred due to lower absorption of oral preparations in alcoholism and malnutrition. Wernicke's encephalopathy has occurred despite high dose vitamin B supplementation orally. ${ }^{29}$ Concerns exist regarding use of parenteral vitamin $B$ preparation after the publication in 1989 by the Committee on Safety of Medicines of a warning about serious adverse reactions relating to Parenterovite. Parenterovite was later withdrawn world wide in 1992 due to manufacturing problems. A new intravenous preparation, Pabrinex, was introduced in 1993. In reality, Parenterovite caused a serious reaction in only one in 250000 cases in the UK and no serious reactions were described in 300000 patients treated with intravenous thiamine in the USA. ${ }^{30}$ These figures pale in comparison with reported allergic reactions in $1 \%-10 \%$ of penicillin administrations and $1 \%-18 \%$ of streptokinase administrations. We routinely administer Pabrinex I and II ampoules diluted in $50-100 \mathrm{ml} \mathrm{5 \%}$ dextrose given over $30 \mathrm{~min}$ utes. This is given twice daily for three days and then patients are switched to oral thiamine $300 \mathrm{mg}$ daily and multivitamins (two daily). One important consideration is that thiamine should be administered before a glucose load in alcoholics with hypoglycaemia as the glucose load may deplete already borderline thiamine stores and thus precipitate Wernicke's encephalopathy.

Patients should be assessed by a dietitian and receive nutritional supplements while very malnourished patients may benefit from nasogastric or nasojejunal feeding.

Patients often require a reducing dose of chlormethiazole or chlordiazepoxide to prevent alcohol withdrawal phenomena. Intravenous chlormethiazole is occasionally used in very agitated patients but should only be used where close cardiorespiratory monitoring is available.

As with other types of liver disease, therapy is indicated for the complications such as hypoglycaemia, ascites, encephalopathy, variceal bleeding, infection, coagulopathy, and renal impairment. When considering encephalopathy in an alcoholic patient, it is important to differentiate this from subdural haematomata, delirium tremens, and Wernicke's encephalopathy.

There is great debate regarding the benefits of corticosteroids in acute alcoholic hepatitis. They inhibit immune mechanisms that cause liver damage as a result of the formation of antibodies to acetaldehyde products. Two meta-analyses suggest that corticosteroids are

\section{Box 9: Management}

- Abstinence: support groups, opiate antagonists, acamprosate

- Vitamins: thiamine, folic acid, and other B vitamins

- Nutrition supplements: nasogastric feeding in malnourished

- Corticosteroids for severe acute alcoholic hepatitis

- Standard treatment for decompensation features such as hypoglycaemia, ascites, varices, infections, encephalopathy, renal failure, and coagulopathy

- Liver transplantation
Box 10: Research treatments
- Propylthiouracil
- Malotilate
- S-adenosyl-L-methionine
- Saturated fatty acids
- Oxandrolone
- Soya bean lecithin
- Thalidomide
- Monoclonal anti-TNF antibodies

beneficial in improving short term survival in patients with severe alcoholic hepatitis. ${ }^{31} 32$ This was confirmed in a randomised, double blind trial comparing 28 days of prednisolone $40 \mathrm{mg}$ daily with placebo in 61 patients with alcoholic hepatitis. At 66 days, mortality in the placebo group was $84 \%$ compared with $12.5 \%$ in the prednisolone treated group. ${ }^{33}$ Corticosteroids should not be given if patients have gastrointestinal bleeding, active infection, pancreatitis, or decompensated diabetes. Otherwise, if patients have severe alcoholic hepatitis in terms of encephalopathy, coagulopathy, leucocytosis, hyperbilirubinaemia or ascites, we treat them with prednisolone $40 \mathrm{mg}$ daily for 28 days. We often judge the severity clinically rather than adhering rigidly to Maddrey's discriminant function score as mentioned previously. Even if short term mortality is reduced, the long term benefits on mortality and prevention of progression to cirrhosis are still unknown.

Propylthiouracil has been proposed as a treatment for ALD because it abolishes the ethanol induced increase in liver oxygen consumption that follows long term ethanol administration. ${ }^{34}$ Initial trials in alcoholic hepatitis produced conflicting results. ${ }^{35}{ }^{36}$ However, a study in 310 patients with chronic liver disease showed that those receiving propylthiouracil (300 mg daily) had a 50\% reduced mortality rate compared with placebo. ${ }^{37}$ Further studies are awaited to confirm these data and therefore the drug is not routinely used in a clinical setting at present. 
Malotilate has been used in the treatment of ALD because it inhibits CYP2E1 induced by alcohol. ${ }^{38} 39$

S-adenosyl-L-methionine dietary supplement therapy has been used in ALD as it replenishes liver mitochondrial glutathione levels. ${ }^{40}{ }^{41} \mathrm{~A}$ defect in transporting glutathione from cytosol into mitochondria caused by ethanol depletes the mitochondrial pool of glutathione. This renders ethanol fed hepatocytes more susceptible to the cytotoxic effects of inflammatory cytokines such as $\mathrm{TNF}-\alpha$ by amplifying the generation of reactive oxygen species within mitochondria.

Saturated fatty acids are protective against ALD. Polyunsaturated fatty acids potentiate alcohol induced liver injury by inducing cytochrome $P-4502 \mathrm{E} 1$. Fatty acid saturation decreases CYP2E1 activity and lipid peroxidation in rat studies. Nanji et al showed that when alcohol feeding was stopped in rats and the dietary fat was switched from fish oil rich in polyunsaturates to palm oil rich in saturated fatty acids, the alcohol induced liver injury was reversed to normal. ${ }^{42}$ When rats were fed fish oil continuously before and after alcohol withdrawal, the liver injury persisted. The role of fatty acids needs to be explored in human ALD.

Long term oxandrolone has been used because of its anabolic effects (improved nitrogen balance) and its ability to accelerate the reversal of the fatty liver of ALD. ${ }^{43}$ It has shown benefit only in moderate disease.

Soya bean lecithin is also a possibility because it can restore phosphatidyl choline levels and inhibit fibrosis in baboons who are fed alcohol. ${ }^{44}$

Thalidomide has been considered in alcoholic hepatitis because it has anti-TNF properties.

\section{Liver transplantation}

Survival after liver transplant for alcoholic cirrhosis is similar to or even better than for other end stage liver diseases. Because of the limited availability of liver transplants, using this option in the management of ALD has become highly emotive because the disease is self inflicted. Most centres demand that patients be abstinent for six months before transplantation. Many candidates are deemed unsuitable because of socioeconomic reasons, psychiatric problems, or coexistent alcoholic damage to other organs such as cardiomyopathy. A major worry is the recidivism rate after transplant. A recent study from Birmingham noted a significant recidivism rate with patients often returning to alcohol intake in the first year after transplant. ${ }^{45}$ These patients rapidly developed histological liver injury including fibrosis.

1 Bellentani S, Saccoccio G, Costa G, et al. Drinking habits as cofactors of risk for alcohol induced liver damage. Gut 1997;41:845-50

2 Becker U, Deis A, Sorensen TIA, et al. Prediction of risk of liver disease by alcohol intake, sex and age: a prospective

3 Kwo PY, Ramchandani VA, O'Connor S, et al. Gender differences in alcohol metabolism: relationship to liver volume and effect of adjusting for body mass. Gastroenterology 1998 115:1552-7.
4 Tanaka K, Tokunaga S, Kono S, et al. Coffee consumtion and decreased serum gamma-glutamyltransferase and aminotransferase activities among male alcohol drinkers. Int 7 Epidemiol 1998;27:438-43.

5 Grant BF, Dufour MC, Harford TC. Epidemiology of alcoholic liver disease. Semin Liv Dis 1988;8:12-25.

6 Teli MR, Day CP, Burt AD, et al. Determinants of progression to cirrhosis or fibrosis in pure alcoholic fatty liver. Lancet 1995;346:987-90.

7 Koskinas J, Kenna JG, Bird GL, et al. Immunoglobulin A antibody to a 200 kilodalton cytosolic acetaldehyde adduct in alcoholic hepatitis. Gastroenterology 1992;103:1060-7.

$8 \mathrm{Xu} \mathrm{D}$, Thiele GM, Beckenhauer JL, et al. Detection of circulating antibodies to malondialdehyde-acetaldehyde adducts in ethanol-fed rats. Gastroenterology 1998;115:68692.

9 Fang C, Lindros KO, Badger TM, et al. Zonated expression of cytokines in rat liver: effect of chronic ethanol and the cytochrome P450 2E1 inhibitor, chlormethiazole. Hepatology 1998;27:1304-10.

10 Neuman MG, Shear NH, Bellentani S, et al. Role of cytokines in ethanol-induced cytotoxicity in vitro in hep G2 cells. Gastroenterology 1998;115:157-66.

11 Polavarapu R, Spitz DR, Sim JE, et al. Increased lipid peroxidation and impaired antioxidant enzyme function is associated with pathological liver injury in experimental associated with pathological liver injury in experimental fish oil. Hepatology 1998;27:1317-23.

12 Fattaccioli V, Andraud E, Gentil M, et al. Effects of ethanol administration on rat liver protease activities: relationship with oxidative stress. Hepatology 1999;29:14-20.

13 Taieb J, Mathurin P, Poynard T, et al. Raised plasma soluble Fas and Fas-ligand in alcoholic liver disease. Lancet 1998;351:1930-1.

14 Hrubec Z, Omenn GS. Evidence of a genetic predisposition to alcoholic cirrhosis and psychosis; twin concordances for alcoholism and its biological endpoints by zygosity among male veterans. Alcohol Clin Exp Res 1981;9:306-9.

15 Grove J, Brown AS, Daly AK, et al. The RSAI polymorphism of CYP2E1 and susceptibility to alcoholic liver phism of CYP2E1 and susceptibility to alcoholic liver dependence on alcohol dehydrogenase genotype. Pharmacogenetics 1998;8:335-42.

16 Grove J, Daly AK, Bassendine MF, et al. Association of a tumor necrosis factor polymorphism with susceptibility to alcoholic steatohepatitis. Hepatology 1997;26:143-6.

17 Day CP, Bashir R, James OF, et al. Investigation of the role of polymorphisms at the alcohol and aldehyde dehyrogenase loci in genetic predisposition to alcohol-related end-organ damage. Hepatology 1991;14:798-801.

18 Wiley TE, McCarthy M, Breidi L, et al. Impact of alcohol on the histological and clinical progression of hepatitis $\mathrm{C}$ infection. Hepatology 1998;28:805-9.

19 Ludwig J, Hashimoto E, Porayko MK, et al. Hemosiderosis in cirrhosis: a study of 447 native livers. Gastroenterology 1997;112:882-8.

20 Grove J, Daly AK, Burt AD, et al. Heterozygotes for HFE mutations have no increased risk of advanced alcoholic liver disease. Gut 1998;43:262-6.

21 MacDonald RA, Jones RS, Pechet GS. Folic acid deficiency and haemochromatosis. Arch Pathol 1965;80:153-60.

22 Rouault TA, Klausner RD. Iron-sulfur clusters as biosensors of oxidants and iron. Trends Biochem Sci 1996;21:174-7.

23 Ganne-Carrie N, Christidis C, Chevret S, et al. Predictive value of liver iron content for survival in 229 prospectively followed patients with cirrhosis. F Hepatol 1996;25(suppl 1):95.

24 Luca A, Carles Garcia-Pagan J, Bosch J, et al. Effects of ethanol consumption on hepatic hemodynamics in patients with alcoholic cirrhosis. Gastroenterology 1997;112:1284-9.

25 Maddrey WC, Biotnott JK, Bedine MS, et al. Coricosteroid therapy of alcoholic hepatitis. Gastrenterology 1978;75:193-

26 Menon DK, Harris M, Sargentoni J, et al. In vivo hepatic $31 \mathrm{P}$ magnetic resonance spectroscopy in chronic alcohol abusers. Gastroenterology 1995;108:776-88

27 Vorobioff J, Groszmann RJ, Picabea E, et al. Prognostic value of hepatic venous pressure gradient measurements in alcoholic cirrhosis: a 10-year prospective study. Gastroenterology 1996;111:701-9.

28 Garbutt JC, West SL, Carey TS, et al. Pharmacological treatment of alcohol dependence. FAMA 1999;281:131825.

29 Chataway J, Hardman E. Thiamine in Wernicke's syndrome- - how much and how long? Postgrad Med 7 1995; $71: 249$

30 Wrenn KD, Slovis CM. Is intravenous thiamine safe? $A m \mathcal{F}$ Emerg Med 1992;10:165.

31 Imperale T, McCullough A. Do corticosteroids reduce mortality from alcoholic hepatitis? Ann Intern Med 1990;113: 299-303.

32 Reynolds T, Benhamou J, Blake J. Treatment of acute alcoholic hepatitis. Gastroenterol Int 1989;2:208-16.

33 Ramond M-J, Poynard T, Rueff B, et al. A randomized trial of prednisolone in patients with severe alcoholic hepatitis. $N$ Engl F Med 1992;362:507-12.

34 Carmichael FJ, Orrego $\mathrm{H}$, Saldivia V, et al. Effect of propylthiouracil on the ethanol-induced increase in liver oxygen consumption in awake rats. Hepatology 1993;18: 415-21.

35 Orrego H, Kalant H, Israel Y. Effects of short term therapy with propylthiouracil in patients with alcoholic liver disease. Gastroenterology 1979;76:105-15. 
36 Halle P, Pare P, Kaptein E, et al. Double blind controlled trial of propylthiouracil in patients with severe acute alco

37 Orrego H, Blake JE, Blendis LM, et al. Long-term treatment of alcoholic liver disease with propylthiouracil. N Engl f Med 1987;317:1421-7.

38 Takase S, Matsuda Y, Yasuhara M, et al. Effects of malotilate treatment on alcoholic liver disease. Alcohol 1989;6: 219-22.

39 Kim SG, Kwak JY, Lee JW, et al. Malotilate, a hepatoprotectant, suppresses CYP2E1 expression in rats. Biophys Res Commun 1994;200:1414-20.

40 Collell A, Garcia-Ruiz C, Miranda M, et al. Selective glutathione depletion of mitochondria by ethanol sensitizes hepatocytes to tumor necrosis factor. Gastroenterology 1998; 115:1541-51.
41 Garica-Ruiz C, Morales A, Colell A, et al. Feeding S-adenosyl-L methionine attenuates both ethanol-induced depletion of mitochondrial glutathione and mitochondrial dysfunction in periportal and perivenous rat hepatocytes. Hepatology 1995;21:207-14

42 Nanji AA, Sadrzadeh SM, Yang EK, et al. Dietary saturated fatty acids: a novel treatment for alcoholic liver disease. Gastroenterology 1995;109:547-54

43 Mendenhall CL, Moritz TE, Roselle GA, et al. A study of oral nutritional support with oxandrolone in malnourished patients with alcoholic hepatitis: results of a Department of Veterans Affairs. Hepatology 1993; 17:564-76.

44 Lieber CS, Robins SJ, Li J, et al. Phosphatidylcholine protects against fibrosis and cirrhosis in the baboon. Gastroenterology 1994;106:152-9.

45 Tang H, Boulton R, Gunson B, et al. Patterns of alcohol consumption after liver transplantation. Gut 1998;43:140-5.

\section{Royal Medical Benevolent Fund}

St Bartholomew's Hospital Choral Society

St Bartholomew's Hospital Choral Society celebrates the millennium and their 35th anniversary with a concert at the Royal Albert Hall on 21 November 2000 in support of their chosen charity for 2000/2001, the Royal Medical Benevolent Fund. The choir will perform Mahler's Symphony No 8, "Symphony of a Thousand" with the Royal Philharmonic Orchestra. Six hundred singers are needed for this monumental work so contact the Barts Choir Honorary Secretary on 01628664157 to participate. To support the Royal Medical Benevolent Fund contact the Fund at 24 King's Road, Wimbledon, London SW19 8QN (tel: 0208540 9194, fax: 0208542 0494, e-mail: rm.bf@virgin.net). 